\title{
Cuestionario de evaluación de la calidad asistencial en un servicio de urgencias urológicas
}

\author{
Martínez-Rodríguez R, Urdaneta Pignalosa G, Rosales Bordes A, Villavicencio Mavrich H.
}

Servicio de Urología. Fundació Puigvert. Barcelona.

Actas Urol Esp. 2008;32(7):717-721

\section{RESUMEN}

CUESTIONARIO DE EVALUACIÓN DE LA CALIDAD ASISTENCIAL EN UN SERVICIO DE URGENCIAS UROLÓGICAS

Introducción: Definida calidad por la propiedad o conjunto de propiedades inherentes a algo, que permiten juzgar su valor, su evaluación se ha convertido en algo obligado en las últimas décadas. La calidad asistencial debe, por tanto, ser inherente al servicio ofrecido. Ha de incluir calidad científica, calidad técnica, gestión y calidad percibida. La evaluación periódica, bien a través de una empresa externa o como auto-evaluación, constituye una forma directa de detectar aspectos susceptibles de mejora.

Material y métodos: Siguiendo los modelos Europeo de Excelencia empresarial (EFQM), la Norma ISO 9001:2000 y el modelo Malcom Baldrige, y partiendo de los indicadores clave del Sistema Nacional de Salud se crea un formulario de evaluación de la calidad global.

Conclusiones: Se propone un cuestionario destinado a evaluar la calidad asistencial de un servicio de urgencias urológicas.

Palabras clave: Calidad. Urgencias. Urología. Asistencial. Gestión.

\section{ABSTRACT}

QUESTIONNAIRE FOR UROLOGICAL EMERGENCY ROOM QUALITY EVALUATION

Introduction: Quality is defined as an essential and distinguishing attribute of something, which allows to evaluate its worth. The quality evaluation has become something necessary during the last years. The assistencial quality should be something inherent to the offered service, including scientific and technical quality, management and quality noticed. A periodical assesment, as auto-evaluation or through an outsourcing, is a recommendable way to detect potential improvement items.

Material and methods: Using the EFQM,ISO 9001:2000 and Malcolm Baldrige model, and through the items from National Health System, a self-questionnaire of urological emergency room quality evaluation is proposed.

Conclusion: A new self-questionnaire of urological emergency room quality evaluation is proposed.

Keywords: Quality. Emergency. Urology. Assistencial. Management.

$\mathrm{D}$ efinida calidad por la propiedad o conjunto de propiedades inherentes a algo, que permiten juzgar su valor, su evaluación se ha convertido en algo obligado en las últimas décadas. La calidad asistencial debe ser inherente al servicio ofrecido. Debe incluir calidad científica, calidad técnica, gestión y calidad percibida. La evaluación periódica, bien a través de una empresa externa o como autoevaluación, constituye una forma directa de detectar aspectos susceptibles de mejora. Se propone un cuestionario destinado a evaluar la calidad asistencial de un servicio de urgencias urológicas.

\section{MATERIAL Y MÉTODOS}

A partir de los indicadores clave del Sistema Nacional de Salud ${ }^{1}$ especialmente de los referidos al sistema sanitario, y siguiendo la estructura de los modelos existentes se crea un formulario de evaluación de la calidad global. Los modelos utilizados son el modelo Europeo de excelencia empresarial (EFQM) ${ }^{2}$, Norma ISO 9001:2000 3 y el modelo Malcom Baldrige ${ }^{4}$.

Desde finales de la década de los '80 surgen modelos destinados a evaluar la calidad. El Malcom Baldrige surge en 1988, y en 1991 nace el modelo de excelencia empresarial (EFGM). La norma ISO 
9001:2000 se enfoca hacia la gestión de la calidad total, recogiendo aportaciones de los modelos previos.

Los modelos de calidad se basan en un concepto de evaluación continuada, basado en las siguientes premisas:

- Concepto de evaluación y mejora continuada. Que en el modelo EFQM viene descrito en la denominada "lógica REDER": Resultados deseadosEnfoque para alcanzarlos-Despliegue de las medidas designadas-Evaluación y Revisión de los resultados.

- Compromiso de la dirección

- Orientado hacia el cliente.

En la Norma ISO 9001:200 la calidad se presupone, y esta frontalmente orientada a garantizar la satisfacción del consumidor. No contempla aspectos de gestión relacionados con recursos humanos (revisión de la efectividad del liderazgo, énfasis del aprendizaje, creatividad e innovación, motivación, apoyo y reconocimiento). Los conceptos de innovación y mejora están más enfatizados en el modelo EFQM.

El modelo EFQM se basa en la premisa: "La satisfacción del cliente y empleado y el impacto en la sociedad se consiguen mediante un liderazgo que impulse la política y la estrategia, la gestión de las personas, los recursos y alianzas y los procesos hacia la conservación de la excelencia en los resultados empresariales".

$\mathrm{El}$ esquema que sigue el modelo EFQM es el siguiente (Fig. 1).

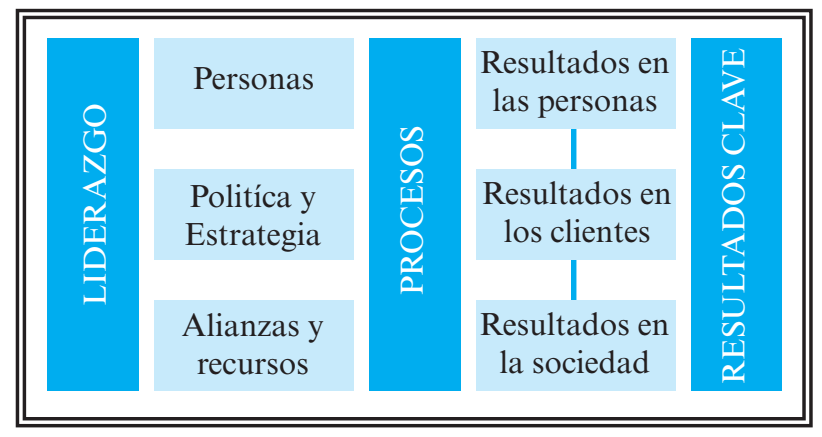

FIGURA 1

Este modelo no es el más idóneo para evaluar un sistema de atención de urgencias, pero si posee una estructura que pueda servir de base para este fin. Modificamos los criterios creando nuevos indicadores.
Alguno de los indicadores claves del Sistema Nacional de salud están incluidos en cuestionarios internacionales de salud, tipo Health Care Quality Indicators Project de OCDE.

En términos generales, un indicador debe reunir dos condiciones ${ }^{5}$ : Importancia y validez científica. Cada una de estas condiciones puede fraccionarse en tres dimensiones:

Importancia:

- Impacto: El indicador debe recoger una situación o actuación que repercuta sobre la salud.

- Importancia política: Debe reflejar aspectos relacionados con el coste de la enfermedad. La base de esta relación coste-enfermedad fue recogida en un estudio pionero ubicado en Canadá ${ }^{6}$.

- Susceptible de ser modificado por el sistema de salud.

Validez científica: Valorada según la:

- Validez interna del indicador

- Validez externa.

- Reproducibilidad.

A partir de la base expuesta creamos un cuestionario destinado a evaluar la calidad del servicio de urgencias de un hospital urológico. De un listado inicial de variables se seleccionan aquellas consideradas más relevantes. Se trata de preguntas cerradas destinadas unas a los profesionales sanitarios y otras a los usuarios, utilizando un cuestionario tipo encuesta como instrumento de medición. El objetivo inicial no es la comparación con otros centros médicos, sino el detectar y desarrollar aquellos puntos susceptibles de mejora.

\section{RESULTADOS}

\section{QUESTIONARIO DE CALIDAD}

\section{Calidad de estructura}

1. Marque la casilla(s) correspondiente(s): El servicio dispone de:

$\square$ Sala de espera

$\square$ Información sobre el tiempo de espera

$\square$ Acceso minusválidos

$\square$ Sistema informático

$\square$ Box individual

$\square$ Material médico especializado.

$\square$ Productos desinfectantes en cada box

$\square$ Sistema de recogida de residuos 


\section{Calidad de servicios}

2. Marque la casilla(s) correspondiente(s): El servicio dispone de:

$\square$ Acreditación docente

$\square$ Especialista de presencia

$\square$ Enfermería especializada

$\square$ Laboratorio de análisis clínico

$\square$ Asistente Social

$\square$ Guías clínicas

$\square$ Unidad de corta estancia

$\square$ Litotricia extracorpórea

$\square$ Acceso a cual(es) de estas exploraciones complementarias:
Ecografía renovesical
Urografía Intravenosa
O Uretrocistoscopia
Tomografía computerizada
Resonancia Magnética Nuclear

\section{Calidad de atención primaria}

3. ¿Cuál es el porcentaje de urgencias atendidas que son readmitidas en las siguientes 72 horas de haber recibido la atención?

$\square$ Menos del 10\%

$\square$ En torno a un 20\%

$\square 50 \%$

$\square 70 \%$

$\square 90 \%$

4. ¿Qué porcentaje de pacientes a los que se indica tratamiento antibiótico son avisados en caso de resistencias antimicrobianas?
$\square>90 \%$
$\square>70 \%$
$\square 50 \%$
$\square<30 \%$
$\square<10 \%$

5. ¿A qué porcentaje de varones diagnosticados de infección urinaria se les ha realizado tacto rectal durante la exploración física?

$$
\begin{aligned}
& \square>90 \% \\
& \square>70 \% \\
& \square 50 \% \\
& \square<30 \% \\
& \square<10 \%
\end{aligned}
$$

6. ¿Qué porcentaje de ocupación de camas del hospital corresponden a ingresos desde urgencias?

$$
\begin{aligned}
& \square<10 \% \\
& \square<30 \% \\
& \square 50 \% \\
& \square>70 \% \\
& \square>90 \%
\end{aligned}
$$

7. ¿Qué porcentaje de la medicación prescrita corresponde a fármacos genéricos?

$$
\begin{aligned}
& \square>80 \% \\
& \square>60 \% \\
& \square>40 \% \\
& \square>20 \% \\
& \square<20 \%
\end{aligned}
$$

8. Indique la(s) asertación(es) correspondientes:

$\square$ A los pacientes se les facilita un informe de alta a la salida del servicio.

$\square$ Existe la posibilidad de realizar litotricia extracorpórea al momento del diagnóstico

$\square$ El servicio participa en proyectos de investigación

$\square$ El servicio interrelaciona con los centros de atención primaria de su área

$\square$ El servicio participa en programas de salud educacional

\section{CALIDAd PERCIBIDA POR El USUARIO}

9. Satisfacción global (Cuestionario a responder por el paciente)

9.1. Considera que la calidad global del servicio es:

$\square$ Excelente

$\square$ Buena

$\square$ Adecuada

$\square$ Insuficiente

$\square$ Muy insuficiente

9.2. Considera que la atención recibida es:

$\square$ Excelente

$\square$ Buena

$\square$ Adecuada

$\square$ Insuficiente

$\square$ Muy insuficiente

9.3. Considera que la información recibida es:

$\square$ Excelente

$\square$ Buena

$\square$ Adecuada

$\square$ Insuficiente

$\square$ Muy insuficiente

10. Gestión de alianzas externas. Relación con el Hospital. (Cuestionario a responder por los otros servicios del hospital).

10.1. La comunicación con el interconsultor es fluida y de fácil acceso: 
$\square$ Totalmente de acuerdo

$\square$ De acuerdo

$\square$ Indiferente

$\square$ En desacuerdo

$\square$ Totalmente en desacuerdo

En referencia a las interconsultas realizadas a este servicio, indique su grado de satisfacción en:

10.2. Tiempo de respuesta:

$\square$ Muy satisfecho

$\square$ Satisfecho

$\square$ Indiferente

$\square$ Insatisfecho

$\square$ Totalmente insatisfecho

10.3. Calidad de respuesta (Resolución de interconsultas):

$\square$ Muy satisfecho

$\square$ Satisfecho

$\square$ Indiferente

$\square$ Insatisfecho

$\square$ Totalmente insatisfecho

\section{Percepción Interna}

Cuestionario a responder por los trabajadores del servicio.

11. Considera que la calidad de atención sanitaria ofrecida por el servicio es:

$\checkmark$ Excelente

$\square$ Buena

$\square$ Adecuada

$\square$ Insuficiente

$\square$ Muy insuficiente

12. Señale su grado de satisfacción personal con el trabajo realizado:

$\square$ Muy satisfecho

$\square$ Satisfecho

$\square$ Indiferente

$\square$ Insatisfecho

$\checkmark$ Totalmente insatisfecho

\section{DISCUSIÓN}

El cuestionario se divide en 5 subgrupos: Calidad de estructura, Calidad de servicios; calidad de atención sanitaria; Calidad percibida por el usuario y percepción interna.
En los dos primeros se evalúa la presencia/ ausencia de una serie de servicios/instalaciones que confieren calidad a un servicio médico. Se evalúa la accesibilidad y la infraestructura de la que el servicio dispone de cara a facilitar la atención al usuario. Los indicadores clave del sistema nacional de salud (SNS), preguntas modificadas del "cuestionario de evaluación sobre calidad de atención al paciente" e interpretaciones sobre los modelos de excelencia empresarial sirven de base a la formulación de dichas asertaciones.

La calidad de atención sanitaria se evalúa mediante 6 preguntas, puntuadas del 1 al 5 las primeras y en forma de puntuación aditiva la última.

La primera hace referencia al número de pacientes readmitidos en las siguientes $72 \mathrm{~h}$ de recibir la atención. Constituye un indicador global de calidad, presentando una leve modificación sobre la "distribución de las urgencias atendidas en los hospitales de cada dependencia según su resolución" que es recogida por el instituto nacional de estadística. A menor porcentaje de pacientes readmitidos mayor puntuación asignada.

La segunda hace referencia al proceso asistencial iniciado y a su seguimiento por parte del centro. Implica un compromiso de idoneidad en el tratamiento asignado. Se evalúa en función del tratamiento de la infección urinaria por ser un proceso frecuente en la consulta de atención urgente e implicar una interrelación con el servicio de microbiología.

La tercera pregunta evalúa la exploración física de los pacientes atendidos. La infección urinaria en varones debe considerarse complicada, y la exploración completa que descarte procesos subyacentes es criterio de calidad.

La cuarta pregunta evalúa la organización del servicio según la ocupación de camas hospitalarias asignadas a la totalidad del servicio. Un número elevado de ingresos puede encubrir la inoperancia de un servicio de urgencias. Los ingresos no han de sobrepasar un porcentaje bajo de las camas disponibles por el centro para no interferir con la programación de ingresos. La disponibilidad de camas asignadas o unidades de corta estancia liberan al resto de los servicios de la carga asistencial de urgencias.

La quinta pregunta recoge la impronta del gasto farmacéutico. La prescripción de genéricos es fomentada 
por el SNS de cara a reducir costes. No toda la prescripción puede basarse en fármacos genéricos, por lo que el corte de calidad se establece en el 60-80\% al ser la mayoría de prescripciones referentes a antibióticos y analgésicos.

En la última cuestión se ofrecen 5 asertaciones evaluadas de forma independiente. Se hace referencia al informe de alta, a la posibilidad de completar el tratamiento con litotricia extracorpórea y a la proyección del servicio en materia de investigación y de interacción social.

La calidad percibida por el usuario no esta incluida en estadísticas internacionales pero si recogida por el SNS. Hace referencia al grado de satisfacción del usuario, de modo individual y representado en los otros servicios del hospital (interconsultas). Se evalúa mediante encuestas que a nivel individual recogen impresiones sobre la atención, la calidad y la información; mientras que intrahospitalariamente recogen la accesibilidad, la rapidez y la resolución.

Finalmente se evalúa la percepción interna. En cualquier empresa de servicios es importante conocer la apreciación propia del trabajo realizado. Los servicios de urgencias se caracterizan por el alto índice de burn-out que sufren sus trabajadores ${ }^{7}$. La detección precoz de la frustración permite motivar al trabajador y evitar su claudicación. Se evalúa mediante dos preguntas orientadas a recoger la impresión de los trabajadores sobre la calidad ofrecida y su grado de satisfacción interno. La primera se orienta a evitar distorsiones entre la idea de lo que se ofrece y lo que el usuario percibe. La segunda busca detectar situaciones de frustración, ya que el nivel de satisfacción personal esta inversamente relacionado con la posibilidad de desarrollar dicho síndrome ${ }^{8}$.

\section{CONCLUSIONES}

Se propone un cuestionario de evaluación orientado hacia la prestación de un servicio, enfatizando la calidad de la atención realizada y la percepción de la misma, tanto por parte del cliente (paciente) como por parte del trabajador sanitario. Se puntúa según una escala de máximo 91, correspondiente a la excelencia asistencial.

\section{REFERENCIAS}

1. Indicadores clave del sistema nacional de salud. http://www. msc.es/organizacion/sns/planCalidadSNS/t01.htm

2. Modelo Europeo de excelencia empresarial http://www.tqm.es/ TQM/ModEur/ModeloEuropeo.htm

3. Norma ISO 9000:2000

http://es.wikipedia.org/wiki/Normas_ISO_9000

4. Modelo Malcom Baldrige http://es.wikipedia.org/wiki/Fundaci \%C3\%B3n_Europea_para_la_Gesti\%C3\%B3n_de_la_Calidad

5. Criteria to select indicator. http://www.oecd.org/dataoecd/1/ 36/36262363.pdf

6. Health Canada,Economic Burden of Illness in Canada, 1988 (Ottawa: Health Canada, 2002).

7. J Sobrequésb; J Cebriáa; J Segurac; C Rodríguez et al. La satisfacción laboral y el desgaste profesional de los médicos de atención primaria" Aten Primaria 2003;31(4):227-33.

8. A. Ozyurt; O.H ayran and H. Sur. "Predictors of burnout and job satisfacction among Turkish physicians”. Q J Med 2006;99:161169.

Correspondencia autor: Dr. R. Martínez Rodríguez Servicio de Urología. Fundació Puigvert.

Cartagena 340-350. 08025-Barcelona. Tel.: 934169700

E-mail autor: hugomar2@yahoo.es

Información artículo: Original - Gestión

Trabajo recibido: noviembre 2007

Trabajo aceptado: abril 2008 